\title{
sciendo
}

\section{Classifying non-banking financial institutions based on their financial performance}

\author{
Adrian COSTEA \\ Bucharest University of Economic Studies, Bucharest, Romania \\ adrian.costea@csie.ase.ro
}

\begin{abstract}
In this paper we evaluate comparatively the performance of non-banking financial institutions in Romania by the means of unsupervised neural networks in terms of Kohonen' SelfOrganizing Maps algorithm. We create a benchmarking model in the form of a two-dimensional map ( $a$ self-organizing map) that can be used to assess visually the performance of non-banking financial institutions based on different performance dimensions, such as capital adequacy, assets' quality and profitability. We use the following indicators: Equity ratio (Leverage) for the capital adequacy dimension, Loans granted to clients (net value) / total assets (net value) for the assets' quality dimension and Return on assets (ROA) for the profitability dimension. We have excluded from our analysis the other three dimensions used in evaluating the performance of banks, due to lack of data (for the two qualitative dimensions: quality of ownership and management) and irrelevance with the NFIs' sector (liquidity). The proposed model is based on the Self-Organising Map algorithm which creates a twodimensional map (e.g. $6 \times 4=24$ neurons) from p-dimensional input data. The data were collected for eleven non-banking financial institutions for four years 2007-2010, in total 44 observations. Using the visualization capabilities of the Self-Organising Map model and the trajectories we show the movements of the three non-banking financial institutions with the worst performance: the largest underperformer denoted with $X$, the second largest underperformer denoted with $Y$ and the third largest underperformer denoted with $Z$ between 2007 and 2010.
\end{abstract}

Keywords: non-banking financial institutions, self-organising maps, performance evaluation, benchmarking, financial ratios

\section{Introduction}

Analysing comparatively financial institutions and being able to accurately predict their performance is a desiderate of all main actors on the financial industry stage. Specifically, regulators of financial industry whose main objective is to maintain the financial stability of the sector are primarly interested in correctly and timely predicting the deterioration of financial institutions' performance. For example, central banks and, especially, their supervision departments are interested in performance prediction models for their supervised entities in order to better allocate their resources (time and personel).

In this paper we employ unsupervised neural networks in terms of Kohonen' SelfOrganizing Maps (SOM) algorithm in order to evaluate comparatively the performance of non-banking financial institutions (NFIs) in Romania. Our objective is to create a benchmarking model in the form of a two-dimensional map (a self-organizing map) that can be used to assess visually the performance of non-banking financial institutions based on different performance dimensions, such as capital adequacy, assets' quality and profitability. 


\section{Literature review}

The research literature in assessing comparatively the companies' financial performance is relatively rich. We include here companies' financial benchmarking, companies' failure prediction, companies' credit/bond rating, analysis of companies' financial statement, analysis of companies' financial text data.

The Self-Organising Map (SOM) algorithm (Kohonen, 1997) has been extensively used in financial applications. For example, Martín-del-Brío \& Serrano Cinca (1993) used SOM to analyze the performance of Spanish banks. The authors used nine financial ratios to discriminate the input space. The dataset consisted of 37 solvent and 29 insolvent banks. The proposed nine financial ratios were as follows: three liquidity ratios: current assets/total assets, (current assets - cash and banks)/total assets, and current assets/loans; three profitability ratios: net income/total assets, net income/total equity capital, and net income/loans; and three other ratios: reserves/loans, cost of sales/sales, and cash flows/loans. The authors constructed a solvency map with different regions of low liquidity, high liquidity, high cost of sales, low profitability, etc. Further, Serrano Cinca (1996) used SOM for predicting the default probabilities of firms. The dataset consisted of five financial ratios that were taken from Moody's Industrial Manual. The time span was 1975 to 1985 and there were 129 firms, of which 64 are solvent and the rest are not. The financial ratios used were the same as in the previous study except the sales/total assets ratio which was eliminated following a preliminary statistical analysis that revealed a poor performance of the ratio in discriminating between solvent and unsolvent companies. Then, the author constructs a new solvency map and revelead the low liguidity, high debt, high profitability, low market values, etc. regions by employing a procedure to automatically extract the solvency clusters. Serrano Cinca (1998a) and Serrano Cinca (1998b) employed SOM to address additional problems, such as: what is the strategy the companies followed in relation to their sector based on their public accounting data, bond valuation problem, and assessing comparatively countris based on their financial and economic performances.

Back et al. (1996), followed by Back et al. (1998), propose SOM as a financial analysis tool. In these studies the authors assessd comparatively the performance of around 120 pulpand-paper companies based on their annual reports for the time interval 1985 to 1989. As Serrano Cinca and his co-authors, Back et al. proposed nine financial ratios, of which four were profitability ratios (operating margin, profit after financial items/total sales, return on total assets, return on equity), one was an indebtedness ratio (total liabilities/total sales), one denoted the capital structure (solidity), one was a liquidity ratios (current ratio), and two were cash flow ratios (funds from operations/total sales, investments/total sales). The authors constructed a SOM (a map) for each year. The individual maps (maps that were displaying only one financial ratio at a time) were used to compare the performance of companies over time: one company trajectory was followed by studying the its position in every fixed-year-map. The authors concluded that SOM is beneficial in identifying and visualizing performance clusters.

Another paper that analyzed the pulp-and-paper industry via a SOM-based model is Eklund et al. (2003). However, here, the authors extended the dataset by gathering data for 77 companies between 1995 and 2000 (6 years). By studying the component plane for each financial ratio, Eklund et al. were able to construct a single SOM for all the years. Then, the authors used the obtained map to show the trajectories of the best and poorest performing companies, and the five largest ones. Another paper (Karlsson et al., 2001) proposed a SOM 
model to study the performance of 88 companies within the telecom industry. The authors proposed seven financial ratios which were collected for five years (1995-1999). Similarly to Eklund et al. (2003), the authors proposed a single SOM for all years and found six financial performance classes. They analyzed the trajectories of the largest telecom companies, Scandinavian companies and countries' averages. In the above two studies, the financial benchmarking was based on financial ratios extracted from the companies' financial statements presented online via their investor relations. Also, the choice of financial ratios is based on the Lehtinen's study of the validity and reliability of ratios in an international comparison (Lehtinen, 1996). Costea \& Eklund (2003) and Costea (2006) applied a combination of self-organising maps and machine learning techniques to classify companies as to their financial performance.

\section{Assessing the performance of non-banking financial institutions}

In Costea (2013) and further in Moinescu \& Costea (2014) we analyzed comparatively the performance of NFIs registered in the Special Register that have financial leasing as main activity and have been active since the introduction of the regulatory framework for these institutions in Romania. In Costea (2013) we analyzed the movements of the three largest (in terms of average total assets for four years 2007-2010) NFIs. Here, we focus on the worst three largest performing companies and check how they evolved over time. The dataset from Costea (2013) consists of the following indicators: Equity ratio (Leverage) for the "capital adequacy" dimension, Loans granted to clients (net value) / total assets (net value) for the "assets' quality" dimension and Return on assets (ROA) for the "profitability" dimension. We have excluded from our analysis the other three dimensions used in evaluating the performance of banks (Cerna et al., 2008), due to lack of data (for the two qualitative dimensions: quality of ownership and management) and irrelevance with the NFIs' sector (liquidity). The data were collected annually for 11 NFIs for four years 2007-2010, in total 44 observations. Costea \& Bleotu (2012) compared the results obtained for SOM with those obtained when a newly proposed fuzzy-logic clustering algorithm was applied on the same dataset.

The proposed model is based on the Self-Organising Map (SOM) algorithm developed by Kohonen in early 80's (Kohonen, 1997). The algorithm creates a two-dimensional map (e.g. $6 \times 4=24$ neurons) from $p$-dimensional input data. After training, each cluster (neuron or unit) of the map contains observations with similar characteristics: companies with similar performance. The SOM topology (the map structure) is either rectangular or hexagonal (the cluster can have four or six neighbours - see Figure 1). Each cluster $i$ is represented by the cluster center (set of weights): $C_{i}=\left\{F R_{i 1}, F R_{i 2}, \cdots, F R_{i p}\right\}$ where, FR represents a financial ratio value.

Before training the SOM, these clusters' centers are randomly initialized (default initialization). The training consists of presenting the observations to the algorithm one by one. The algorithm calculates the closest cluster for each observation and this cluster is called the best matching unit $\left(\mathrm{m}_{\mathrm{u}}\right)$. If we denote by $\mathrm{O}_{\mathrm{j}}$ a particular observation, then $\mathrm{m}_{\mathrm{u}}$ is given by the cluster center for which the Euclidean distance is minimized:

$m_{u}=\left\{C_{i} \mid \min \left(\sqrt{\sum_{k=1}^{p}\left(O_{j k}-C_{i k}\right)^{2}}\right)\right\}$ 


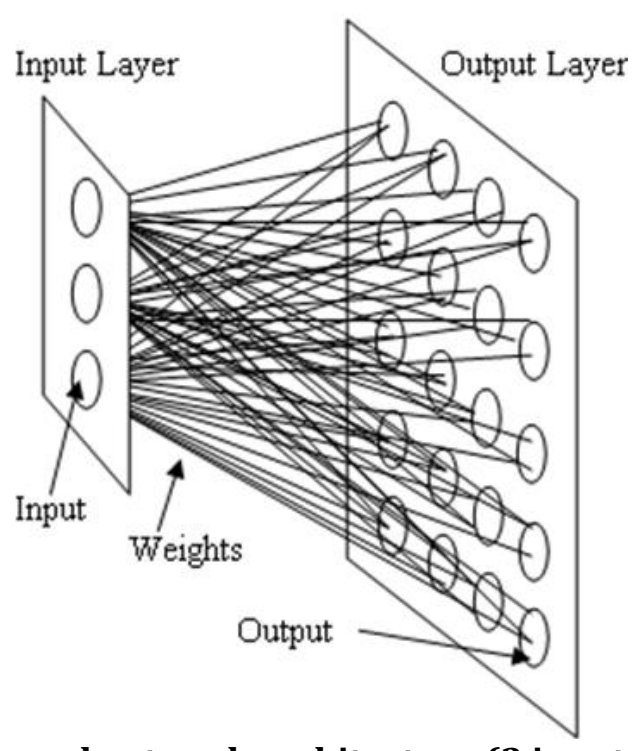

Figure 1: Example of SOM neural network architecture (3 inputs and 5x4 rectangular map) Source: Costea (2005)

Once we find the best matching unit for a particular observation, the center of that winning cluster $u$ is changed by incorporating the new won observation. Also, the neighbouring clusters change their centers:

$C_{i}(t+1)=\left\{\begin{array}{ll}C_{i}(t)+\alpha(t)\left[O_{j}-C_{i}(t)\right], & i \in N_{u} \\ C_{i}(t) & , i \notin N_{u}\end{array}\right\}$

where $\mathrm{O}_{\mathrm{j}}$ is the randomly selected observation at moment $t, \mathrm{~N}_{\mathrm{u}}$ is a set of clusters in the vicinity of the winner neuron $u$ and $0<\alpha(t)<1$ is the learning rate. The $\alpha(\mathrm{t})$ parameter decreases monotonically with $t$ (Kohonen, 1997, pp. 86-88, Alhoniemi et al., 1999, pp. 4-5). The learning rate $\alpha(t)$ can be a linear function (the default):

$\alpha(t)=\alpha(0)(1-t / r l e n)$

or a inverse-type function:

$\alpha(t)=\alpha(0) C /(C+t)$

where $\alpha(0)$ is the initial learning rate, $\mathrm{C}=100 /$ rlen and rlen is number of steps in training. In any case, $\alpha(\mathrm{t})$ decreases to 0 . The set $\mathrm{N}_{\mathrm{u}}$ can be defined using the radius length $N$ (the radius of the circle, which represents the vicinity of the winner $u$ ). $N$ can be defined as a decreasing function of time:

$N(t)=1+[N(0)-1](1-t / r l e n)$

where $N(0)$ is the initial radius length. $\mathrm{N}(\mathrm{t})$ decreases linearly to 1 .

The algorithm stops when a predefined number of training steps has been reached (the default stopping criterion) or if the improvement in the overall average quantisation 
error $(E)$ is very small. The overall average quantisation error is given by formula:

$E=\sqrt{\frac{\sum_{j=1}^{n} \Sigma_{k=1}^{p}\left(o_{j k}-C_{i k}\right)^{2}}{n}}$

where $\mathrm{C}_{\mathrm{i}}$ is the winner cluster $u$ (the cluster that wins the observation). It is the cluster closest to the observation $\mathrm{O}_{\mathrm{j}}$.

The final output of SOM training is a matrix with the set of clusters' centers (weight vectors). Ultsch (1993) proposed a method called $U$-matrix method to visualize the SOM output (the weights matrix). The unified distance matrix (U-matrix) method computes all distances between neighbouring clusters. The borders between neurons are then constructed on the basis of these distances: dark borders correspond to large distances between two neurons involved, while light borders correspond to small distances (see the bottom of Figure 2). In this way we can visually group the neurons ("raw" clusters) that are close to each other to form supra-clusters ("real” clusters). In Figure 2 the „real“ clusters are bordered with a dashed line. In addition to the U-matrix map, a component plane or feature plane can be constructed for each individual financial ratio (input variable). In the feature planes light/"warm" colours for the neurons correspond to high values, while dark/"cold" colours correspond to low values (see the top of Figure 2).

Taking into account the advantages of $U$-matrix method, we proceed in two stages: in the first stage we apply the SOM algorithm and build the 2-dimensional map from the input space. For the first stage of the methodology we have trained several maps by selecting different values for the above SOM algorithm parameters. We used our dataset without preprocessing the data given the values of the ratios are already standardised in a $[-1 ; 1]$ interval. In this way the potential negative impact the Euclidean distance calculations would have on the clustering is diminished. The right choice of the SOM parameters is problem and data-dependent. However, whatever data we use for training, the algorithm works best when the results of a first training session are calibrated by a second training session where the parameters are fine-tuned (Kohonen et al., 1996, p. 16). The final map chosen (shown on the bottom of Figure 2) was the best in terms of quantisation error $(E=0.074522)$. The map dimensionality was set to $6 \times 4$ (24 "raw" neurons or clusters) and not higher since we do not want to obtain too many empty clusters. We randomly initialized the clusters' centers and choose a hexagonal map structure. We used equation (3) as the learning rate function and equation (5) as the neighborhood function. Next, as reported elsewhere, we used two training phases: in the first training phase (rough training) the clusters' centers are ordered. Consequently, we used higher initial learning rate and higher initial vicinity: $r l e n=1000, \alpha(0)$ $=0.05$, and $\mathrm{N}(0)=6$. In the second training phase the clusters' centers are fine-tuned. Therefore, we used lower learning and neiborhood parameters, but higher number of training steps: rlen $=10000, \alpha(0)=0.02$, and $\mathrm{N}(0)=2$.

The final 6x4 map contains 24 "raw" clusters or neurons (in Figure 2, a "raw" cluster is any of the 24 hexagons in the map). After the training, each neuron contains a number of observations or none. We used the final map obtained in Costea (2013), shown in Figure 2. 


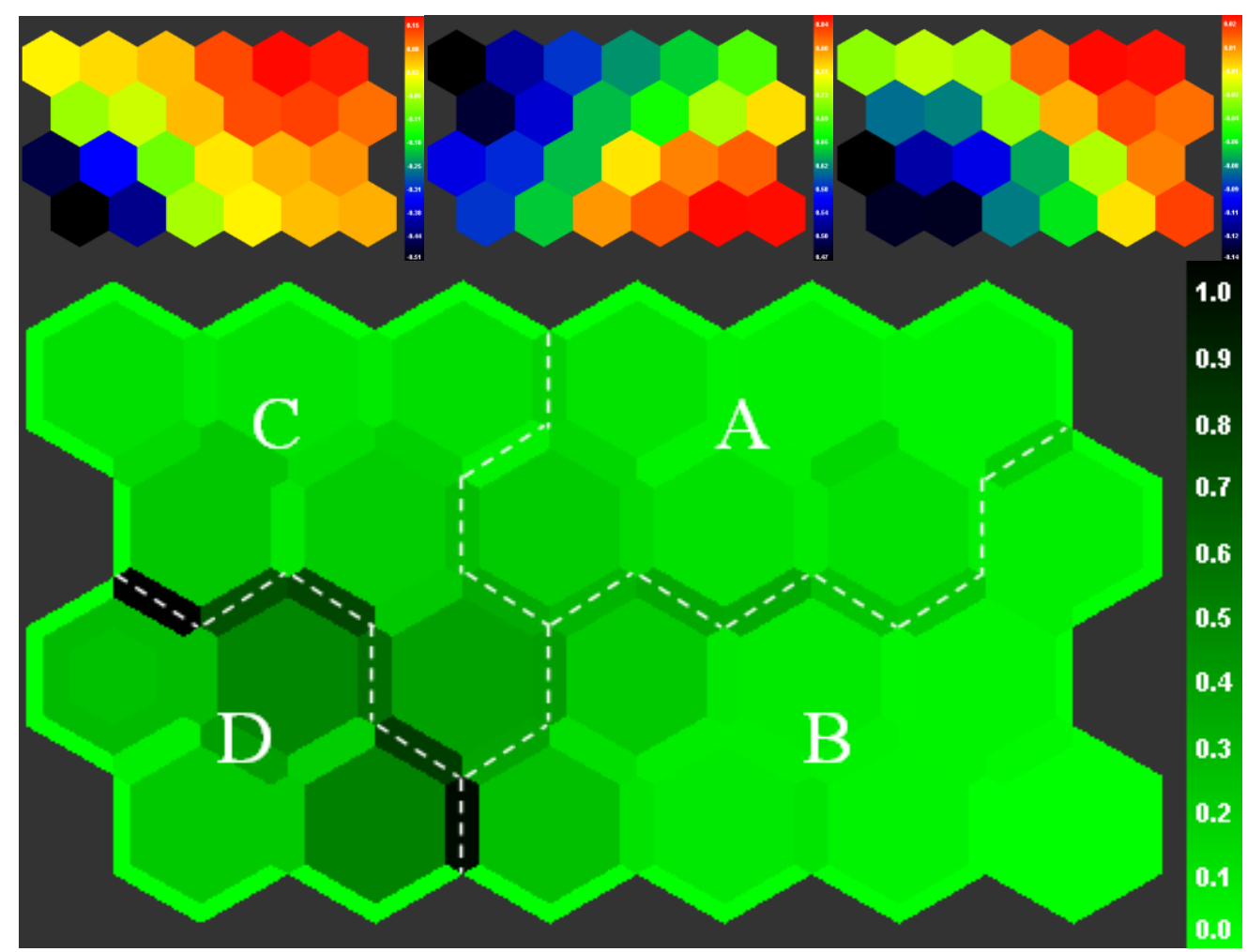

Figure 2. The SOM based on NFIs performance dataset: 24 "raw" clusters and 4 identified "real" clusters. On the top of the figure we display the component planes for the 3 variables: Leverage, Loans/Asstes and ROA

Source: adapted from Costea (2013)

The second stage of the methodology consists of grouping the "raw" clusters to form "real clusters". This is done by using the U-matrix method (looking at the borders between neurons), by analysing the component plane for each input variable and the observations that belong to each cluster. In this way we have identified 4 "real" clusters (clusters A, B, C, and D in Figure 2) which are described as follows.

Cluster A includes the NFIs with the highest values for the input variables measuring capital adequacy and profitability and medium values registered for the variable measuring the assets' quality. This "real" cluster contains 8 observations. The average values for the variables are: Leverage $-14.77 \%$, Loans/Assets $-63.48 \%$, ROA $-0.88 \%$. It is the only cluster with positive average profitability ratios.

Cluster B is the largest cluster containing half of the total observations (22 observations). It includes NFIs with medium capital adequacy and profitability and highest value for the variable measuring assets' quality. The average values for the variables are: Leverage - 2.98\%, Loans/Assets - 81.13\%, ROA - (2.24\%).

All ratios in cluster $C$ have average values: Leverage $-1.39 \%$, Loans/Assets $-53.23 \%$, ROA - (2.50\%). However, this cluster contains NFIs with a lower performance than those in cluster B. Both cluster B and C contain NFIs that in average have negative profitability ratios.

Cluster D contains the worst performers. All performance ratios show low values: Leverage - (52.15\%), Loans/Assets - 57.33\%, ROA - (13.24\%). Again, the profitability ratios are negative in average.

Next, in Figure 3 we analyse the movements of the NFIs that presented the worst 
performance (they have been classified in one of the four years in cluster D). We denote these NFIs with Company X, Y and Z in decreasing order of average assets (X - 687,298,550 RON, Y - 521,088,420 RON, and Z - 148,508,941 RON).

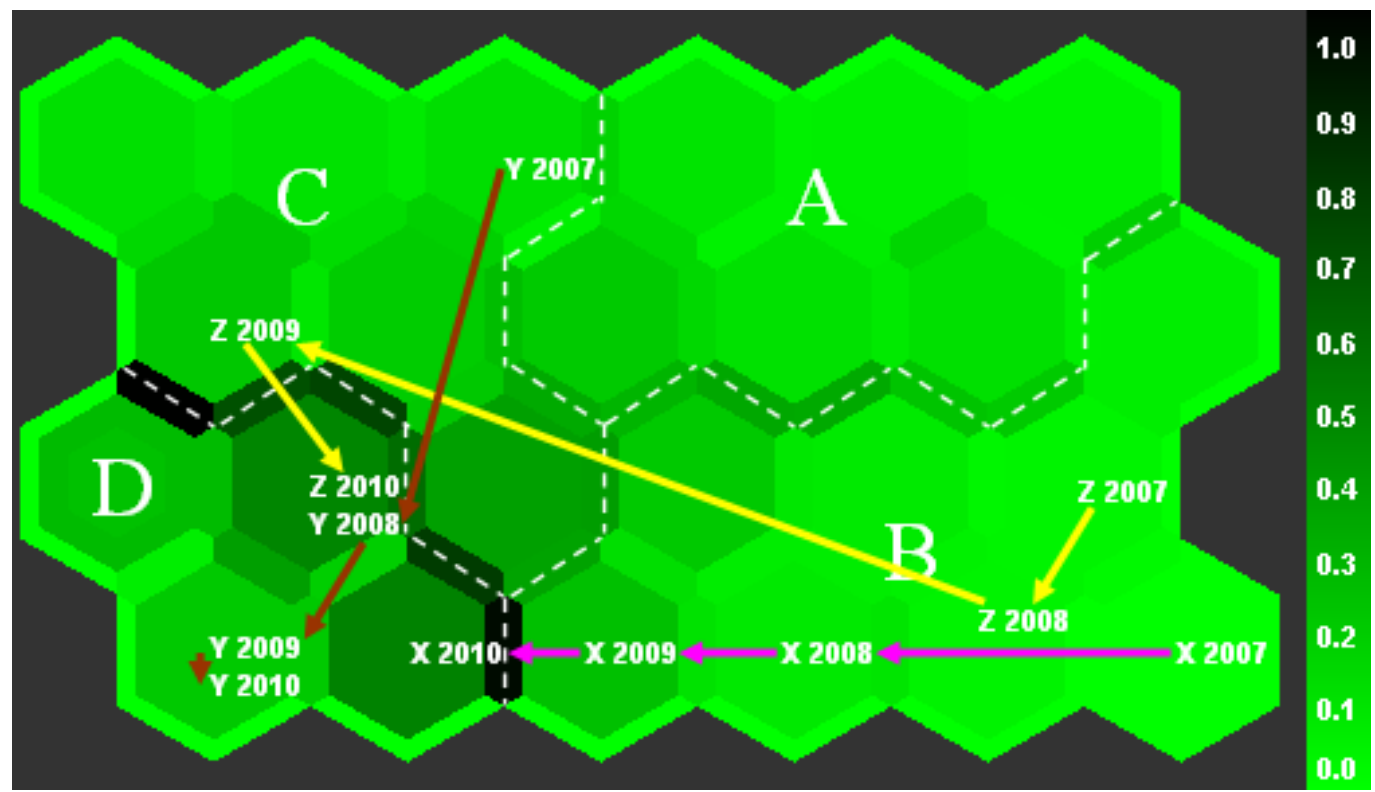

Figure 3. The final 6x4 map with identified "real" clusters and the trajectories between 2007 and 2010 for the worst performers ("pink" for company $X$, "brown" for company $Y$, and "yellow" for company Z)

The trajectories in Figure 3 show the movements of the three NFIs with the worst performance: the largest underperformer denoted with $X$ (pink), the second largest underperformer denoted with $Y$ (brown) and the third largest underperformer denoted with $\mathrm{Z}$ (yellow) between 2007 and 2010.

Company X started in cluster B in 2007 and 2008, and remained there in 2009. In the next year the company' situation deteriorated and it dropped to cluster D. This was partially due to constant deterioration of its own capital with the highest deterioration encountered for the year 2010 as compared to smaller decrease in total assets. At the same time, in 2010 the loans granted by company X have decreased dramatically as compared to 2007 , reaching almost a $65 \%$ decrease.

The second largest underperformer NFI, company Y, showed the lowest values for all performance indicators during the analysis period. It started in cluster C in 2007, and it dropped in cluster D where it remained for the following three years. Company $\mathrm{Y}$ encountered the worst capital adequacy (-90\%) mainly due to a sharp decrease of its total assets (61\% decrease in 2010 as compared to 2007). In 2007, 2008 and 2009, company Y encountered negative values for the profitability ratio (ROA), with the highest value encountered for 2009. In 2010, the profitability ratio turned positive due to company's restructuring measures.

Company Z had a similar evolution with company X: it started in cluster B in 2007 and 2008, passed to cluster $C$ one year later and, finally, dropped to cluster D in 2010. Like company X, in 2010 company Z encountered a significant decrease in all its performance indicators: high negative figure for the own capital and a dramatic loan decrease of about 
$70 \%$ as compared to 2008. However, the ratios decrease was slightly smaller than the decrease in absolute values as the denominator (total assets) also decreased substantially (by about 45\%).

\section{Conclusions}

This paper presented a neural-network-based model to evaluate comparatively the performance of non-banking financial institutions (NFIs) in Romania. Based on the SOM algorithm we were able to build four performance classes. We used quarterly data for 11 NFIs, the time span being 2007-2010 period, and for a set of NFIs' performance indicators (employed to capture three most important performance dimensions: capital adequacy, assets' quality and profitability). We analyzed the three worst performer NFIs by using the visualization capabilities of SOM model and trajectories.

\section{References}

Alhoniemi, E., Hollmen, J., Simula, O., \& Vesanto, J. (1999). Process Monitoring and Modeling Using the Self-Organising Map. Integrated Computer-Aided Engineering, 6(1), 3-14.

Back, B., Sere, K., \& Vanharanta, H. (1996). Data Mining Accounting Numbers Using Self Organising Maps. In J. Alander, T. Honkela, M. Jakobsson (eds.), Proceedings of Finnish Artificial Intelligence Society Conference, 35-47. Vaasa, Finland.

Back, B., Sere, K., \& Vanharanta, H. (1998). Managing Complexity in Large Databases Using Self-Organizing Maps. Accounting Management and Information Technologies, 8(4), 191-210.

Cerna, S., Donath, L., Seulean, V., Herbei, M., Bărglăzan, D., Albulescu, C., \& Boldea, B. (2008). Financial Stability. Timișoara: West University Publishing House.

Costea, A. (2005). Computational Intelligence Methods for Quantitative Data Mining. TUCS PhD dissertation No. 67, Åbo Akademi University, Turku, Finland.

Costea, A. (2006). The Analysis of the Telecommunications Sector by the Means of Data Mining Techniques. Journal of Applied Quantitative Methods (JAQM), 1(2), 144-150.

Costea, A. (2013). Performance benchmarking of non-banking financial institutions by means of Self-Organising Map algorithm. East-West Journal of Economics and Business, XVI(1), 37-58.

Costea, A., \& Bleotu, V. (2012). A new fuzzy clustering algorithm for evaluating the performance of non-banking financial institutions in Romania. Economic Computation and Economic Cybernetics Studies and Research, 46(4), 179-199.

Costea, A., \& Eklund, T. (2003). A Two-Level Approach to Making Class Predictions. In RH. Sprague Jr. (ed.), Proceedings of $36^{\text {th }}$ Annual Hawaii International Conference on System Sciences (HICSS 2003) (9 pages). IEEE Computer Society, Hawaii, USA, January 6-9, 2003, Track: Decision Technologies for Management, Minitrack: Intelligent Systems and Soft Computing, ISBN: 0-7695-1874-5.

Eklund, T., Back B., Vanharanta, H., \& Visa, A. (2003). Financial Benchmarking Using SelfOrganising Maps - Studying the International Pulp and Paper Industry. In J. Wang J. (ed.), Data Mining - Opportunities and Challenges (Chapter 14 - pp. 323-349). Hershey, PA: Idea Group Publishing.

Karlsson, J., Back, B., Vanharanta, H., \& Visa, A. (2001). Financial Benchmarking of Telecommunications Companies. TUCS Technical Report, No. 395.

Kohonen, T. (1997). Self-Organising Maps (2nd ed.). Heidelberg: Springer-Verlag.

Kohonen, T., Hynninen, J., Kangas, J., \& Laaksonen, J. (1996). SOM_PAK: The Self-Organising Map Program Package. Helsinki University of Technology, Report A31, Otaniemi.

Lehtinen, J. (1996). Financial Ratios in an International Comparison. Validity and Reliability. Acta Wasaensia 49, Vaasa, Finland.

Martín-del-Brío, B., \& Serrano Cinca, C. (1993). Self Organizing Neural Networks for the 
Analysis and Representation of Data: some Financial Cases. Neural Computing \& Applications, Springer Verlag (ed.), 1(2), 193-206.

Moinescu, B., \& Costea, A. (2014). Towards an early-warning system of distressed nonbanking financial institutions. Economic Computation and Economic Cybernetics Studies and Research, 48(2), 75-90.

Serrano Cinca, C. (1996). Self Organising Neural Networks for Financial Diagnosis. Decision Support Systems, 17, 227-238.

Serrano Cinca, C. (1998a). SOM as a tool for Initial Data Analysis (Let Financial Data Speak for Themselves). In G. Deboeck, T. Kohonen (eds.), Visual Intelligence in Finance: withg Self-organising Maps (pp. 4-17). Berlin: Springer Verlag.

Serrano Cinca, C. (1998b). From Financial Information to Strategic Groups - a Self Organising Neural Network Approach. Journal of Forecasting, 17, 415-428.

Ultsch, A. (1993). Self organized feature maps for monitoring and knowledge aquisition of a chemical process. In S. Gielen, B. Kappen (eds.), Proceedings of the International Conference on Artificial Neural Networks (ICANN93) (pp. 864-867). London: SpringerVerlag. 